\title{
Editorial: The 2020 François Naftali Frenkiel Award for Fluid Mechanics
}

The recipients of the 37th François Naftali Frenkiel Award for Fluid Mechanics are Matthew Butler, Finn Box, Thomas Robert, and Dominic Vella for their paper "Elasto-capillary adhesion: Effect of deformability on adhesion strength and detachment" which was published in Physical Review Fluids 4, 033601 (2019). They were presented with this award on November 24, 2020 at the annual meeting of the APS Division of Fluid Dynamics held as a virtual meeting. Each author received a check for $\$ 500$ plus a scroll acknowledging the award. The Frenkiel Award citation reads: "The paper was selected for its insightful and elegant combination of analytical models and experiments that lead to novel understanding of the physical mechanisms that control elasto-capillary adhesion."

The purpose of this Award, which is named after Dr. F. N. Frenkiel, is to recognize significant contributions in fluid mechanics by young investigators. The Award is sponsored by the Division of Fluid Dynamics of the APS and, beginning with the 2017 award, has been given annually to a young author of a paper that has been published in Physical Review Fluids, during the calendar year preceding the presentation. "Young" will normally be defined as having had not more than twelve years of full-time employment after their most advanced academic degree was awarded prior to the year of the paper's publication. More than one author may be involved, and one author may be a thesis advisor sharing the Award if he/she also meets the same twelve year requirement. The winner of this Award is selected by a committee appointed by the Chair of the Division of Fluid Dynamics of the APS.

François Naftali Frenkiel was born in Warsaw, Poland on September 19, 1910, and received his Ph.D. in Physics from the University of Lille in France, where he studied under the direction of Kampé de Fériet. He came to the U.S. in 1947 and was associated successively with Cornell University, the U.S. Naval Ordnance Laboratory, the Johns Hopkins University Applied Physics Laboratory, and, from 1960 until his retirement, with the David W. Taylor Naval Ship Research and Development Center. In addition to being the founder and longtime editor of Physics of Fluids, he served on a large number of national and international committees, including the Division of Fluid Dynamics of the American Physical Society, of which he was the chairman and secretary on numerous occasions. $\mathrm{He}$ published extensively in the field of turbulent flows and pioneered the application of high-speed digital computing methods to the measurement of turbulence and the mathematical modeling of urban pollution. Dr. Frenkiel retired in 1981 and passed away on July 9, 1986, in Washington, DC. 

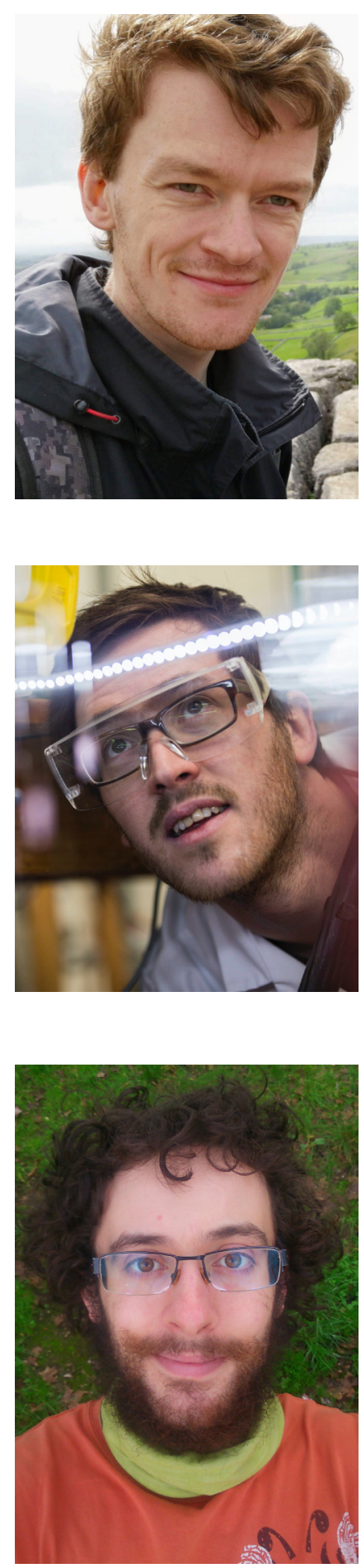

Matt Butler received his DPhil in Mathematics at the University of Oxford, working on mathematical models of capillary adhesion, and is now a postdoctoral researcher in microscale soft active matter at the University of Birmingham. He is interested in problems involving viscous and free-surface flows, fluid-structure interactions and microfluidics.

Finn Box is now a post-doctoral researcher in the Gulliver Laboratory of the ESPCI Paris. He enjoys using laboratory experiments to explore flows, fluid-structure interactions and elastic instabilities. Finn received his Ph.D. from the University of Manchester and has also worked as a post-doc in the Universities of Cambridge, Oxford and Manchester.

Thomas Robert performed some of the experimental work reported in the paper during an internship in Oxford as part of his Masters' degree at ENS, Paris. His interests are broad and include joining a project to redesign the blast furnace around sustainable principles. Ultimately, he plans to become a Physics and Chemistry teacher and hopes to teach in international locations in which education is not widely accessible. 


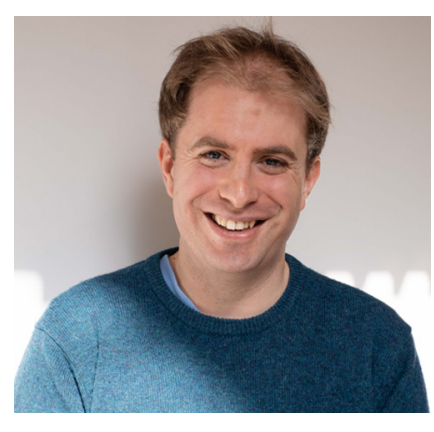

Dominic Vella is a Professor of Applied Mathematics in the Mathematical Institute at the University of Oxford. He studied Mathematics at Trinity College, Cambridge, before a post-doc split between the ENS and ESPCI, Paris. His current research is focused on the instability of slender elastic objects, including wrinkling and snap-through. $\mathrm{He}$ is particularly interested in situations where these instabilities are driven by fluid loading and/or capillary effects.

John Kim

Gary Leal

Editors

(2) Published 21 January 2021

DOI: 10.1103/PhysRevFluids.6.010001 